\title{
Inhalt
}

Vorwort VII

\section{Elisabeth Breidt}

Neuartige Wörterbücher für Mensch und Maschine: Wörterbuchdatenbanken in COMPASS

Ian F. Roe

Collins Cobuild Valency Dictionary: The Patterns of English Verbs, Adjectives and

Nouns: Zum Verfassen eines Lernerwörterbuches .27

\section{Martha Ripfel}

Bedeutungserlăuterungen im allgemeinen einsprachigen und im Textwörterbuch am

Beispiel des Redensartenwörterbuchs zur Fackel.

Stefan J. Schierholz

Zur Semantik der Prăposition ,auf“" in komplexen Nominalphrasen sowie notwendigen und moglichen Darstellungen im Wörterbuch

Angelika Storrer

Hypermedia-Wörterbücher: Perspektiven für eine neue Generation elektronischer

Wörterbücher

Ingrid Lemberg

Lexikographische Erläuterungen im Deutschen Rechtswörterbuch: Gestaltungsmuster

in einem Worterbuch der alteren deutschen Rechtssprache

Jochen A. Bär

Vorschläge zu einer lexikographischen Beschreibung des frühromantischen Diskurses

Wolfgang Müller

Worterbücher der Zukunft - oder: Terrae incognitae

Ingrid Lemberg / Sybille Petzold / Heino Speer

Der Weg des Deutschen Rechtswörterbuchs in das Internet.

Fritz Hermanns

Kausative Adjektive. Bericht über eine lexikologisch-lexikographische Recherche .289 
Klaus Mudersbach

Ein Vorschlag zur Beschreibung von Phrasemen auf der Basis eines universalen pragmatischen Modells

Herbert Ernst Wiegand

Altes und Neues zur Makrostruktur alphabetischer Printwörterbücher

Anhang: Abstracts und Résumés 373

Namenregister.....

Sachregister. 389 